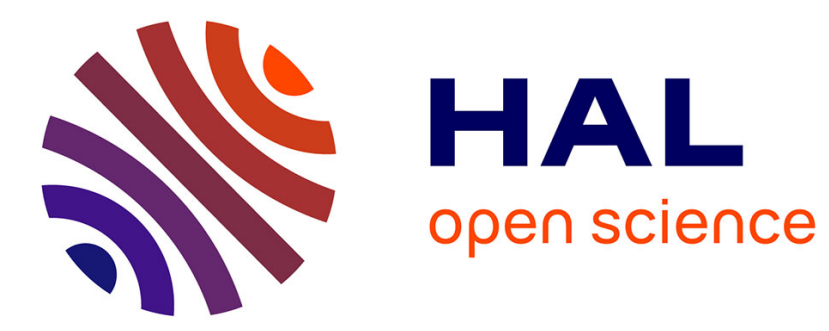

\title{
Pour une écriture multimédia de l'ethnomusicologie
}

Marc Chemillier

\section{To cite this version:}

Marc Chemillier. Pour une écriture multimédia de l'ethnomusicologie. Journées d'Informatique Musicale, Jun 2003, Montbéliard, France. hal-02994154

\section{HAL Id: hal-02994154 \\ https://hal.science/hal-02994154}

Submitted on 7 Nov 2020

HAL is a multi-disciplinary open access archive for the deposit and dissemination of scientific research documents, whether they are published or not. The documents may come from teaching and research institutions in France or abroad, or from public or private research centers.
L'archive ouverte pluridisciplinaire HAL, est destinée au dépôt et à la diffusion de documents scientifiques de niveau recherche, publiés ou non, émanant des établissements d'enseignement et de recherche français ou étrangers, des laboratoires publics ou privés. 


\title{
Pour une écriture multimédia de l'ethnomusicologie
}

\author{
Marc Chemillier
}

Les possibilités techniques offertes par le développement du multimédia ouvrent aujourd'hui de nouvelles perspectives pour «donner à voir» les musiques du monde. Plusieurs réalisations significatives existent déjà, concernant les musiques de tradition orale, mais aussi d'autres répertoires musicaux, sous la forme de cédéroms ou de pages web ${ }^{1}$. Elles préfigurent sans doute d'autres contenus plus développés qui alimenteront, à l'avenir, des dispositifs audio-visuels de plus grande envergure installés dans des espaces de projection publics, par exemple au sein des musées. Dans cette optique, il est intéressant d'étudier l'usage qui est fait des nouvelles possibilités techniques dans les réalisations multimédia existantes, et de dégager quelques propriétés spécifiques des modes d'écriture auxquels elles donnent naissance dans le champ de l'ethnomusicologie.

Les figures illustrant cette présentation sont empruntées à une série d'animations musicales interactives, appelées clés d'écoute, développées sur le site web ethnomus.org à l'initiative du Laboratoire d'ethnomusicologie du Musée de l'Homme. Les premiers exemples de ce corpus, réalisés avec les moyens du bord, avaient été présentés aux journées de la SFE à Royaumont en avril 2000. La réalisation d'une nouvelle série d'animations plus complexes fût confiée en janvier 2002 aux soins d'une équipe de professionnels du multimédia réunie par Annick Armani, à laquelle ont participé Ingrid Guichard, Pascal Joube et Flavie Jeannin. Ces nouvelles clés d'écoute furent montrées lors d'une journée organisée au Cube d'Issy-lesMoulineaux par Annick Armani en mars 2002, avec le soutien de la SFE, sur le thème de l'écriture multimédia pour l'ethnomusicologie et de ses implications dans la communication scientifique. Jean-Claude Gardin participait à ces journées, auxquelles il apporta la profondeur de sa réflexion épistémologique. Les clés d'écoute ont ensuite été présentées aux journées de la SFE de Carry-le-rouet en mai 2002, ainsi que dans diverses occasions à l'extérieur du cercle des ethnomusicologues, en particulier au séminaire de l'Ina-GRM consacré à l'apport du multimédia à l'analyse musicale ${ }^{2}$. Le site web ethnomus.org est conçu comme un laboratoire permettant d'expérimenter diverses formes d'écriture multimédia pour l'éthnomusicologie, en vue de la publication de cédéroms d'anthologie et de la création de contenus multimédia pour des musées.

Les premières clés d'écoute du site ethnomus.org (sur la musique de harpe Nzakara ou les rondes funéraires Toraja) étaient de simples schémas, analogues à ceux qui accompagnent habituellement les textes des ethnomusicologues, à cela près qu'ils avaient été sonorisés et animés. La technologie multimédia consistait principalement à intégrer le son et l'image, à souligner des parties de l'image, et à déplacer des éléments dans l'animation, pour attirer l'attention du spectateur sur certains aspects. Somme toute, le multimédia reprenait à son compte des procédés déjà utilisés dans le film et la vidéo. Plus récemment est apparu pour nous l'intérêt d'une innovation beaucoup plus spécifique de la technologie multimédia : la possibilité de faire interagir l'utilisateur avec l'image et le son en cliquant sur divers composants de l'interface visuelle qu'il a sous les yeux. Dès lors, il devient nécessaire de se demander sur quoi il est intéressant de faire agir un utilisateur par rapport au sens d'une musique, afin de donner à l'écriture multimédia une portée scientifique qui dépasse les visées

\footnotetext{
1 On trouvera un échantillon de ces réalisations dans les références citées en fin d'article.

2 L'intervention d'Annick Armani à ce séminaire est partiellement disponible en ligne sur le site de l'Ina.
} 
pseudo-ludiques dans lesquelles restent parfois confinées les réalisations multimédias qui sont marquées par le modèle des jeux vidéos. Dans les clés d'écoute les plus récentes du site ethnomus.org (sur le chant diphonique ou les polyphonies vocales de Sardaigne), la réflexion sur l'interactivité a donné naissance, comme on le verra dans cet article, à de véritables scénarios interactifs conçus comme des sortes de «démonstrations », dont l'ambition est de synthétiser par les moyens de l'écriture multimédia une parcelle de connaissance ethnomusicologique.

\section{Intégration du son et des images animées}

L'image permet de représenter le flux sonore. Les ethnomusicologues connaissent bien cette possibilité, qu'ils utilisent depuis les débuts de leur discipline pour transcrire les musiques de tradition orale. De nombreux types de transcriptions ont été utilisés : la notation musicale usuelle sur portées, le sonagramme pour visualiser le spectre acoustique, différentes formes de tablatures pour codifier les doigtés, et bien d'autres modes de représentations plus spécifiques adaptés à tel ou tel répertoire. L'intégration d'une transcription dans un objet multimédia permet différentes sortes de traitements. Avant d'aborder l'interactivité, qui sera étudiée dans la section suivante, nous allons dans cette section envisager les trois traitements élémentaires suivants :

1) le soulignage de certaines parties de la transcription,

2) la sonorisation de l'image en synchronisant image et son,

3) l'animation d'éléments de l'image.

Lorsque la musique est transcrite sous forme d'image, l'une des premières possibilités offertes par la représentation graphique est de souligner un élément de la transcription pour attirer l'attention du lecteur. Cette possibilité est d'ailleurs indépendante de l'intégration multimédia. Par exemple, sur une simple feuille de papier à musique, on peut entourer un motif mélodique dans une transcription en notation musicale.

Ensuite, l'intégration dans un média unique du son et de l'image introduit une dimension nouvelle essentielle qui est la synchronisation image-son. On peut en effet sonoriser l'image, en synchronisant la représentation de la musique avec la musique elle-même, comme le permet également le film ou la vidéo. Ces deux premiers traitements, synchronisation imageson et soulignage de parties de l'image, sont les deux aspects sur lesquels reposent les «musicographies » développées par le GRM pour la représentation multimédia des musiques électroacoustiques ${ }^{3}$.

Le multimédia présente enfin une troisième dimension à travers l'animation graphique, qui offre une possibilité supplémentaire de capter l'attention du spectateur. En effet, une image ne produit pas le même effet si elle est donnée à voir en entier d'un seul coup (comme le sont les

\footnotetext{
${ }^{3}$ Le GRM a développé un logiciel, appelé Acousmographe, qui permet de « retoucher » le sonagramme d'une séquence musicale grâce à une palette d'objets graphiques. On peut ainsi souligner certaines zones du spectre, en y incrustant des motifs graphiques représentant des objets musicaux, et de cette manière mettre en évidence certains aspects de la forme musicale. Les images obtenues (qui, au-delà de leur rôle de représentation de la musique, sont souvent de belles images ayant des qualités graphiques propres) peuvent évidemment être regardées en écoutant la séquence sonore correspondante synchronisée avec l'image. En général, plusieurs images sont associées à une même séquence sonore, et traduisent ce que François Delalande appelle des «points de vue » sur l'œuvre représentée. Des objets multimédias de ce type, appelés «musicographies », sont publiés dans le cédérom Musique électroacoustique du GRM et sur divers sites web à vocation pédagogique, ainsi que dans le cédérom de l'équipe du MIM à Marseille, qui adapte la notion de musicographie à des œuvres nonélectroacoustiques, en particulier instrumentales.
} 
musicographies), ou si elle se construit progressivement sous les yeux du spectateur en plusieurs étapes successives. Dans ce cas, elle peut produire dans l'esprit du spectateur une illumination, provoquer un déclic mental en révélant (au sens où une photographie se « révèle » lorsqu'elle est plongée dans le bain du révélateur) un aspect de la musique passé inaperçu ou une propriété musicale spécifique.

Un bel exemple de cette utilisation de l'animation comme révélateur se trouve dans le «gamelan mécanique » de la Cité de la musique. Réalisé en collaboration avec Catherine Basset sous forme d'animation multimédia sur le site web cite-musique.fr, pour servir de support pédagogique à la préparation du programme de l'option musique du baccalauréat 2003 traitant des musiques de Bali et Java, ce gamelan mécanique permet d'écouter des exemples de musique de gamelan associés à divers modes de visualisation. L'un d'eux est une photographie des instruments de l'orchestre gamelan, dans laquelle chaque fois qu'un gong ou une touche de métallophone est frappée, son image sur la photographie devient lumineuse, et la lumière s'estompe progressivement avec la résonance de l'instrument. Cet effet joliment réalisé permet au spectateur de suivre la musique du gamelan en repérant les différentes sources sonores.

Dans le même ordre d'idées, le rôle de révélateur joué par l'image animée intervient dans plusieurs exemples des clés d'écoute du site ethnomus.org. Mais contrairement au gamelan, où l'utilisation de l'animation ne met en évidence aucune propriété particulière de la musique (le même effet aurait été obtenu avec une simple séquence filmée montrant les musiciens en train de jouer le gamelan, l'œil pouvant alors suivre les gestes des instrumentistes), les animations du site ethnomus.org révèlent certains aspects de la musique qui ne sont pas accessibles par la seule observation de gestes instrumentaux.

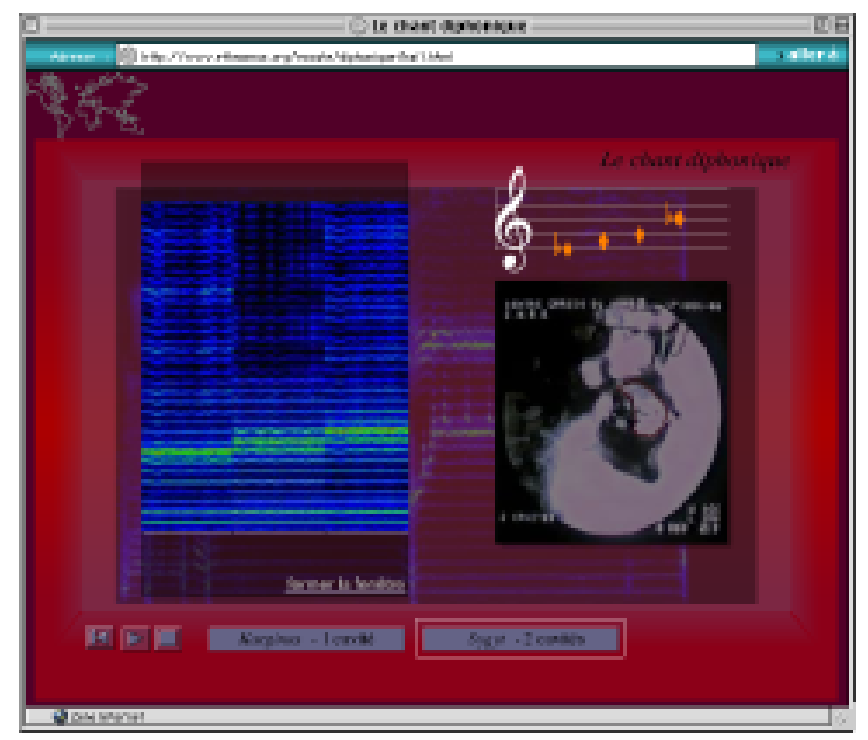

Figure 1. Chant diphonique (Tran Quang Hai) : aspects physiologiques de la technique vocale

L'animation sur le chant diphonique est inspirée du film Le chant des harmoniques réalisé par Hugo Zemp en collaboration avec Tran Quang Hai. L'une des innovations majeures de ce film est l'utilisation de radiographies pour expliciter l'aspect physiologique de la technique vocale du chant diphonique. Il est ainsi possible de mettre en évidence le rôle de la langue dans la division de la cavité buccale en deux parties, et la sélection des harmoniques par un 
déplacement entre l'avant et l'arrière. L'image de la Figure 1 souligne les contours de la cavité buccale antérieure (entre la langue et les dents), et l'animation montre les déformations de cette cavité permettant de produire les quatre notes transcrites sur la portée au-dessus de la radiographie.

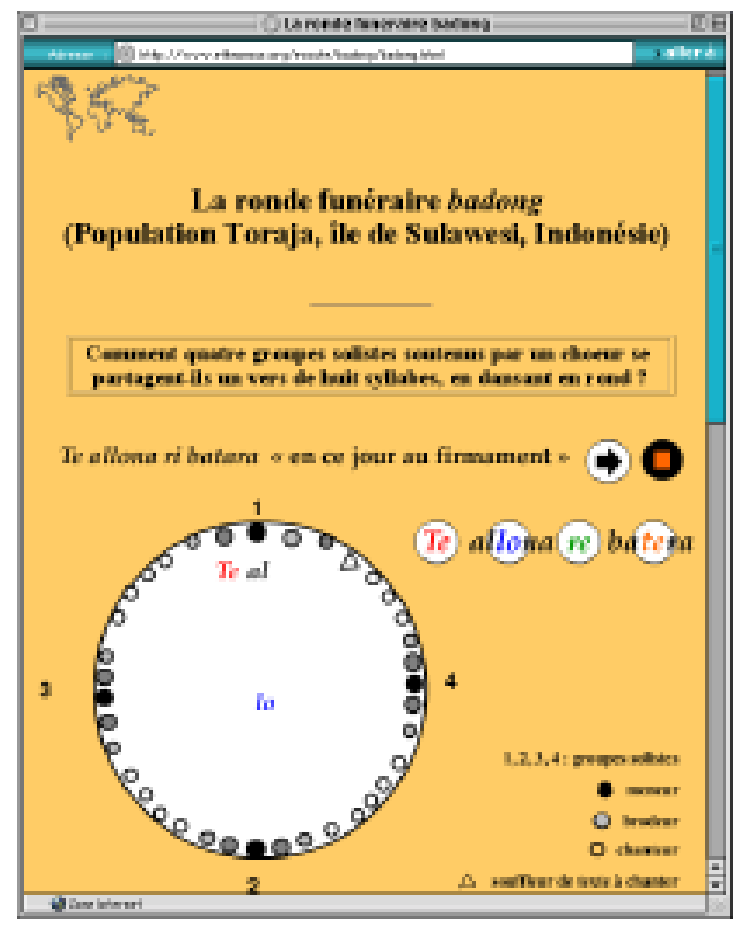

Figure 2. Rondes funéraires Toraja (Dana Rappoport) :

partage des syllabes d'un vers à l'intérieur d'un chœur disposé en cercle

Dans la clé d'écoute consacrée aux rondes funéraires Toraja d'Indonésie, c'est le mode de spatialisation du son qui est explicité par l'animation. Le chœur disposé en cercle est représenté en vue de dessus. Les chanteurs sont répartis selon quatre groupes opposés deux à deux, et chantent en alternance les syllabes d'un vers qui se trouve ainsi «partagé » entre les groupes. L'animation permet de suivre le déplacement des syllabes entre les quatre groupes. Dans la Figure 2, la syllabe lo est transmise du groupe 1 (au nord de la figure) au groupe 2 (au sud).

Enfin, la clé d'écoute sur la musique de harpe Nzakara révèle la forme remarquable de certains ostinati de harpe joués par les poètes-harpistes. Les cordes étant pincées par couples, la formule de harpe comporte deux lignes mélodiques superposées, l'une sur les cordes graves, l'autre sur les cordes aiguës. Grâce à l'image animée, on peut suivre simultanément les profils de ces deux lignes qui se dessinent à l'écran progressivement et constater qu'ils sont identiques à un décalage près, ce qui confère à cet ostinato de harpe une forme apparentée au canon. 


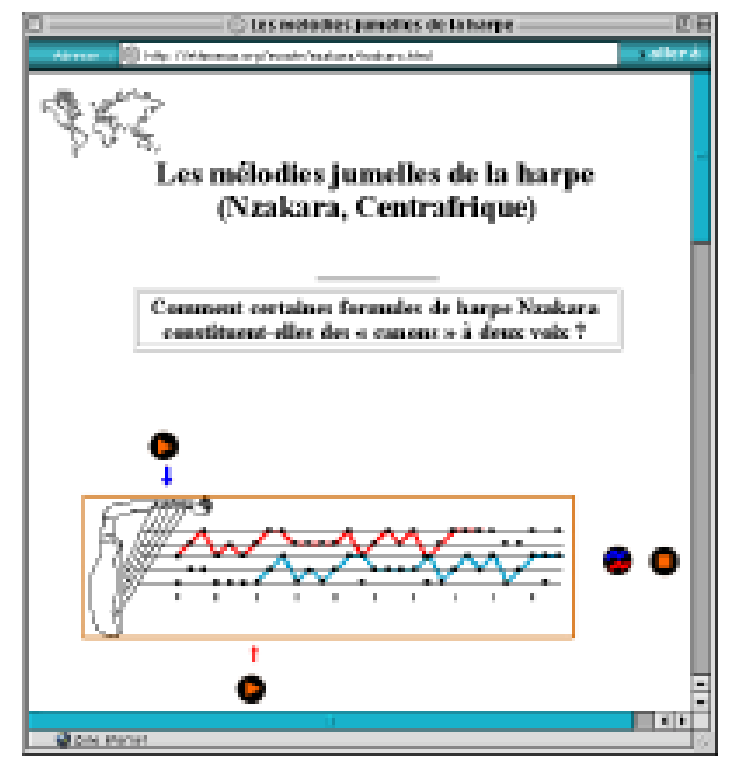

Figure 3. Musique de harpe Nzakara (Marc Chemillier) : deux lignes mélodiques de mêmes profils

\section{Choix des points d'interaction}

Toucher l'objet multimédia, en agissant sur les données sonores, est une expérience qui contribue à modifier la perception que l'on a de la musique. Cette possibilité est introduite lorsque l'on passe du film ou de la vidéo à l'objet multimédia interactif. Ce que nous appelons ici interactivité, c'est la possibilité pour l'utilisateur d'intervenir dans le déroulement de l'animation pour modifier ce qu'il voit et ce qu'il entend, à travers un dispositif d'interface adapté (simple clic de souris dans le cas d'un ordinateur, capteurs gestuels plus complexes s'il s'agit d'une animation interactive projetée dans une salle, par exemple à l'intérieur d'un musée), et de ce fait, de procéder à différentes formes d'expérimentation sur le répertoire musical qui lui est présenté.

La conception d'animations musicales interactives traitant des musiques de traditions orales doit donc aborder le problème suivant : sur quoi est-il intéressant de faire agir l'utilisateur par rapport au sens d'une musique? De nombreuses manières d'interagir avec les données musicales sont possibles, mais toutes ne contribuent pas de façon intéressante à l'écriture d'un discours ethnomusicologique. Celles qui le font doivent éclairer l'utilisateur sur le sens de la musique étudiée, dans le contexte de la société où elle est produite. Le choix des points d'interaction est donc essentiel dans la conception d'animations musicales interactives à contenu ethnomusicologique. En définitive, ces points peuvent être considérés comme autant de clés pour écouter ces musiques d'une autre manière, d'une manière culturellement déterminée. Les deux types d'actions les plus intéressants qui ont été mis en pratique jusqu'à aujourd'hui sont, à notre point de vue, d'une part la séparation des voix dans les musiques fondées sur l'intrication de parties polyphoniques ou polyrythmiques complexes, et d'autre part la sélection d'une composante dans un spectre harmonique pour les musiques fondées sur le renforcement de certains harmoniques, comme le chant diphonique ou les polyphonies de Sardaigne.

Séparer les voix d'une polyphonie renvoie à la technique bien connue du re-recording que Simha Arom avait introduite dans les années soixante-dix en pratiquant sur le terrain des enregistrements multipistes. Il était donc naturel que de tels enregistrements en voix séparées 
soient utilisés dans le cédérom consacré aux Pygmées Aka, qu'il a publié en collaboration avec Suzanne Fürniss et une équipe d'anthropologues. On peut ainsi écouter plusieurs extraits de musiques Aka en activant ou en désactivant à sa guise les voix de la polyphonie, et en effectuant toutes les combinaisons imaginables de ces différentes voix, ce qui permet en quelque sorte de démêler l'enchevêtrement polyphonique et polyrythmique. C'est une expérience d'écoute très enrichissante, qui modifie profondément la perception que l'on a de cette musique. Une technique analogue d'activation/désactivation des voix d'une polyphonie est utilisée dans le gamelan mécanique de la Cité de la musique, permettant de percevoir les différentes vitesses de rotation des parties instrumentales autour du gong central qui marque le retour de chaque cycle ${ }^{4}$. Enfin, dans la clé d'écoute consacrée à la musique de harpe Nzakara déjà citée (Figure 3), on peut écouter séparément les deux lignes mélodiques constituant le « canon », et ainsi se persuader auditivement de l'identité de leurs profils.

L'autre type d'action utilisé dans les clés découte est la sélection d'une ou plusieurs composantes du spectre harmonique. Cette opération est rendue possible grâce au logiciel Audiosculpt (développé à l'Ircam), qui permet d'afficher le sonagramme d'un extrait sonore, de gommer certaines parties du spectre, et de recalculer par un procédé de synthèse additive le signal sonore correspond au spectre modifié. Elle est très utile pour dévoiler le mécanisme intime des musiques fondées sur le renforcement de certaines zones spectrales. Sur le site ethnomus.org, nous l'avons utilisée dans les clés d'écoute consacrées au chant diphonique, et aux polyphonies vocales de Sardaigne.

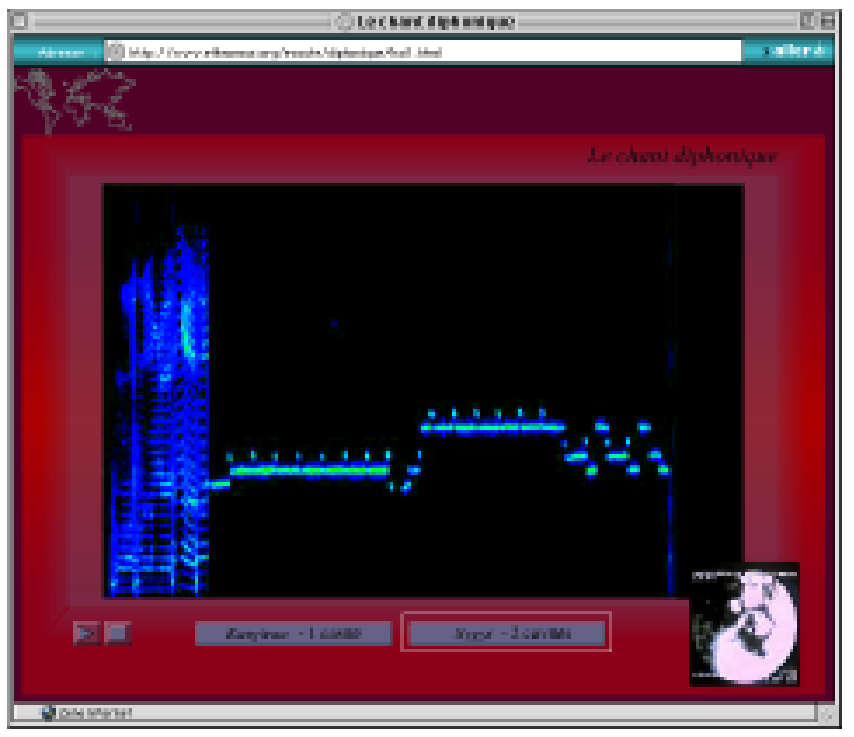

Figure 4. Chant diphonique (Tran Quang Hai) : sélection de la mélodie harmonique

Dans l'animation sur le chant diphonique déjà citée, on peut en effet écouter soit l'enregistrement original, soit la mélodie harmonique seule. Le passage de l'un à l'autre facilite la perception de cette mélodie, en permettant à l'utilisateur de se repérer dans le spectre

\footnotetext{
${ }^{4}$ Il faut noter que la reconstitution d'une polyphonie à partir des voix séparées ne donne pas le même résultat acoustique que l'enregistrement de la polyphonie elle-même. C'est le prix à payer pour permettre à l'utilisateur de combiner librement les voix séparées. On s'en rend compte dans le cas du gamelan, où l'écoute de la superposition des voix séparées fait perdre l'impression de halo sonore que produit habituellement cette musique, sans doute parce qu'il manque certaines qualités sonores de résonance sympathique qui apparaissent quand les instruments sont joués ensemble.
} 
harmonique, et de localiser mentalement la mélodie. La même technique est utilisée dans l'animation interactive consacrée aux polyphonies vocales de Sardaigne, comme on le verra dans la section suivante.

\section{Scénarisation et raisonnement logique}

Au-delà de l'expérience isolée consistant à séparer les voix d'une polyphonie, ou à sélectionner des composantes dans un spectre harmonique, une animation interactive permet aussi de coordonner une série d'expériences succcessives, constituant une progression logiquement organisée. On peut en effet construire un parcours interactif sur le modèle d'un raisonnement, comme on va le voir dans cette section. L'architecture logique d'un scénario interactif, l'enchaînement des expériences proposées à l'utilisateur, peuvent suivre la progression d'une véritable argumentation de sciences humaines, c'est-à-dire une succession d'arguments dont le déroulement concourt à établir certaines propriétés de l'objet étudié. Cette approche permet d'éviter l'écueil du gadget «presse-bouton », de la production multimédia dans laquelle l'interactivité est réduite à des visées pseudo-ludiques, où l'utilisateur potentiel est considéré comme un personnage un peu immature qu'il s'agirait de divertir.

Le modèle de cette approche du scénario interactif est l'animation conçue par Bernard LortatJacob sur les polyphonies vocales de Sardaigne. Nous allons décrire les étapes du parcours imaginé par Bernard Lortat-Jacob, sous la forme d'une visite virtuelle dans les « salles » d'un musée métaphorique, chaque salle étant constituée d'un écran comportant différents boutons d'action et correspondant à un chapitre du scénario.

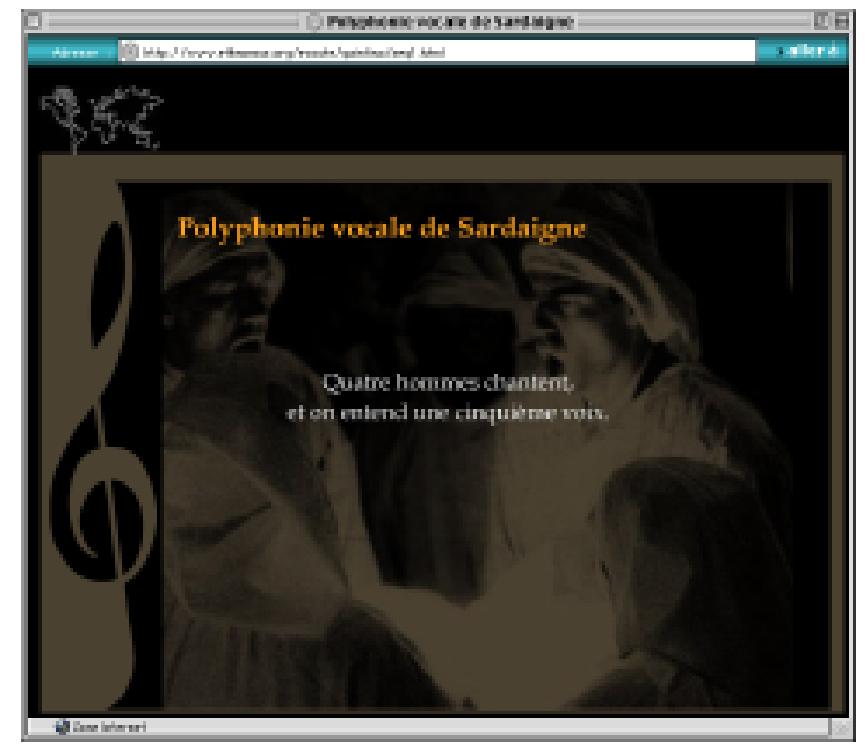

Figure 5. Polyphonies de Sardaigne (Bernard Lortat-Jacob) : écran introductif

L'animation commence par une petite séquence animée introductive, non sonore, conduisant d'abord à une phrase en forme d'énigme, qui résume le problème abordé par l'animation (Figure 5), « quatre hommes chantent et on entend une cinquième voix. », puis se terminant par une page de texte présentant succinctement le répertoire, avec lien vers l'écoute de la transcription d'un extrait.

La première « salle » de la visite est consacrée à la représentation traditionnelle de la musique 
sous forme de transcription solfégique. On a la possibilité d'écouter un extrait de deux minutes qui permet d'apprécier le déploiement de cette musique dans le temps pendant une durée significative, en suivant la partition découpée en quatre pages d'écrans successifs. Pendant l'écoute, un bouton apparaît au-dessus des quatre portées, avec un commentaire, qui ouvre la porte de la salle suivante.

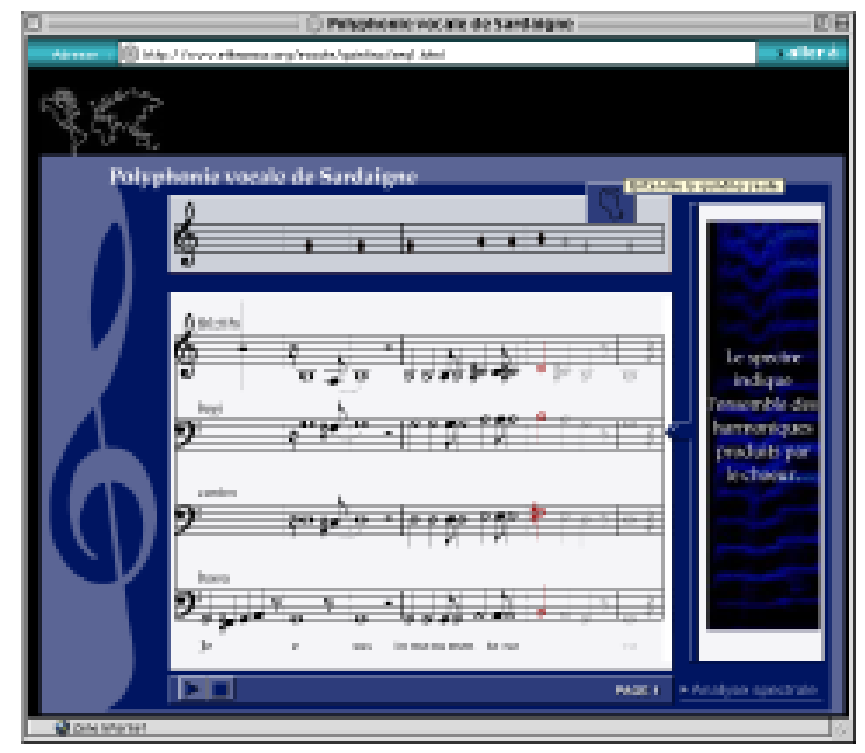

Figure 6. Polyphonies de Sardaigne (Bernard Lortat-Jacob) : écoute de la cinquième voix isolée

L'étape suivante de la visite donne à l'utilisateur la possibilité de réécouter un fragment de trente secondes, en basculant entre deux modes, l'un consistant à écouter la polyphonie complète, l'autre consistant à écouter la cinquième voix seule isolée par Audiosculpt, selon un procédé analogue à celui décrit dans la section précédente pour le chant diphonique. Cette expérience perceptive permet de localiser mentalement dans le spectre la cinquième voix fusionnelle appelée quintina. Une portée supplémentaire apparaît au-dessus des autres matérialisant cette cinquième voix non chantée (Figure 6).

Le chapitre trois du scénario propose une représentation plus technique de la musique, sous forme de sonagramme, et met à la disposition de l'utilisateur la même expérience d'écoute que précédemment sur l'extrait de trente secondes, avec polyphonie complète ou quintina isolée. Une gomme tracée sur le sonagramme sert de pointeur vers l'étape suivante du scénario. 


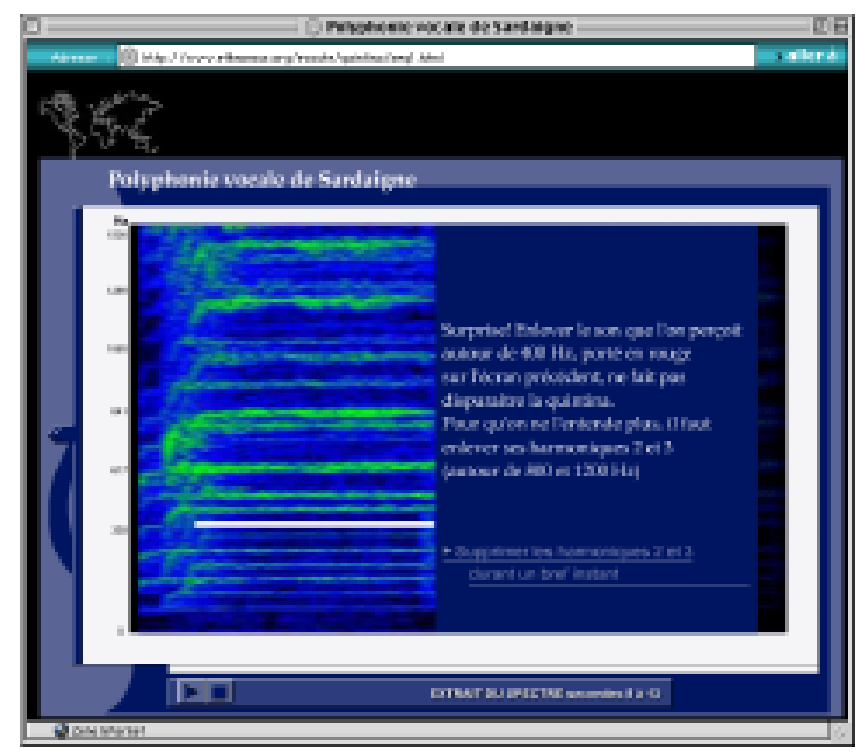

Figure 7. Polyphonies de Sardaigne (Bernard Lortat-Jacob) : suppression de la cinquième voix dans le spectre

Dans la salle qui suit, on propose à l'utilisateur de gommer la quintina sur une partie du spectre (correspondant à un accord de dix secondes prélevé dans l'extrait précédent). La partie effacée apparaît d'abord en surbrillance à la hauteur de $400 \mathrm{~Hz}$ où l'on perçoit la quintina, puis elle est supprimée du spectre. On réécoute le passage, et surprise ! La quintina est toujours là. Un panneau explicatif apparaît pour dévoiler la clé de l'énigme (Figure 7).

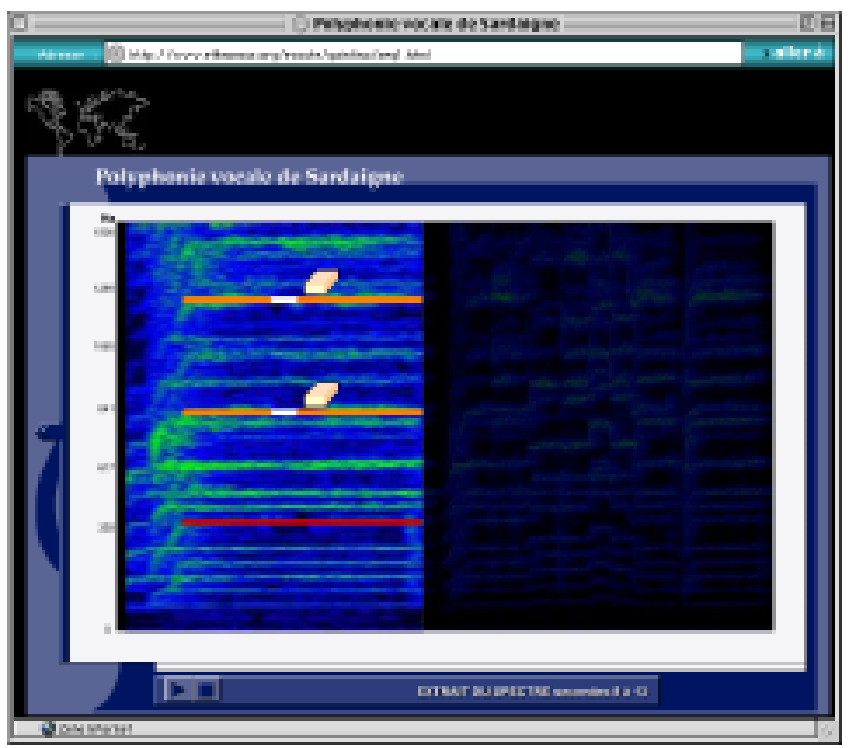

Figure 8. Polyphonies de Sardaigne (Bernard Lortat-Jacob) : suppression des harmoniques 2 et 3 de la quintina

La visite se termine par une dernière expérience. On propose à l'utilisateur d'enlever non pas la quintina elle-même, mais ses harmoniques 2 et 3 à environ 800 et $1200 \mathrm{~Hz}$ respectivement. On le fait sur un petit fragment à l'intérieur de l'extrait de dix secondes précédent. L'expérience permet d'entendre clairement la disparition, puis la réapparition de la quintina. La visite s'achève avec un panneau conclusif, qui résume la thèse exposée dans cette animation : la quintina est une voix fusionnelle obtenue par la superposition de plusieurs 
harmoniques renforcées dans le spectre des chanteurs (Figure 8).

\section{Conclusion}

La technologie multimédia offre à l'ethnomusicologie des possibilités techniques susceptibles de bouleverser en profondeur les pratiques de communication scientifique en usage dans cette discipline. Le film et la vidéo avaient déjà depuis longtemps tiré parti de la possibilité de synchroniser une image avec du son, et de souligner certains aspects dans une représentation graphique de la musique. Plus récemment, la possibilité d'interagir avec un objet multimédia a ouvert la voie à de nouvelles expériences perceptives permettant de guider mentalement un auditeur vers certains aspects importants de la musique écoutée.

Dans une approche plus ambitieuse, l'écriture multimédia touche à la question de l'argumentation scientifique elle-même. Les expériences menées sur le site ethnomus.org ont montré qu'il est possible de matérialiser un raisonnement scientifique à travers la scénarisation d'une animation musicale interactive. D'autres expériences ont été menées dans l'utilisation de structures hypertextuelles, c'est-à-dire des structures constituées de textes reliés les uns aux autres en cliquant sur des liens, pour représenter l'architecture d'un discours de sciences humaines selon le modèle de la schématisation logiciste décrit par Jean-Claude Gardin ${ }^{5}$. Il est d'ailleurs envisageable d'associer les deux approches. Quelque soient les voies explorées, l'utilisation du multimédia en ethnomusicologie, et la définition d'une véritable écriture multimédia pour cette discipline, sont indissociables d'une réflexion épistémologique qui devrait conduire les chercheurs à repenser la manière dont il organisent leurs idées à propos des musiques qu'ils étudient.

\section{Références}

CHEMILlIER M., RAPPOPORT D., Pourquoi présenter des modèles musicaux sur Internet?, Actes de la table ronde Sémantique et Archéologie : aspects expérimentaux. Renouvellements méthodologiques dans les bibliothèques numériques et les publications scientifiques, organisée par l'Ecole française d'Athènes, Athènes, 18 et 19 novembre 2000, édités par Andrea Iacovella, Bulletin de Correspondance Hellénique (à paraître).

GARDIN J.-C., LAGRANGE M.-S., MARTIN J.-M., MOLINO J., NATALI J., La logique du plausible : essais d'épistémologie pratique [en sciences humaines] (Paris, Maison des sciences de l'homme, 1981).

Cédéroms

AROM S., BAHUCHET S., EPELBOIN A., FÜRNISS S., GUILLAUME H., THOMAS J., Pygmées Aka. Peuple et musique, Paris, Montparnasse Multimédia, 1998.

BESSON D., Les musicographies, Ina-GRM R 9501, 1995.

DONNIER P., Flamenco-soft, Prix Moëbius international 1997 (catégorie culture), non

\footnotetext{
5 Le cédérom réalisé en archéologie par Valentine Roux et Philippe Blasco illustre cette approche. En ethnomusicologie, Dana Rappoport prépare un cédérom qui s'inscrit dans la même démarche, et qui comprendra une base de données d'enregistrements et de documents de terrain, une arborescence inspirée du logicisme pour représenter la construction théorique résultant des recherches ethnomusicologiques qu'elle a effectuées sur ce répertoire, et enfin une série de clés d'écoute (comme celle de la figure 2) explicitant certains aspects musicaux particuliers du répertoire.
} 
publié.

GRM, La musique électroacoustique, Ina-GRM, Collection Musiques tangibles 1, Paris, Hyptique, 2000.

MIM, Les unités sémiotiques temporelles, nouvelles clés pour l'écoute, Marseille, 2002.

ROUX V., BLASCO P., Cornaline de l'Inde, livre-cédérom (Paris, Maison des Sciences de l'Homme, 2000).

Internet

ARMANI A., Présentation de l'animation sur les polyphonies vocales de Sardaigne, InaGRM, séminaire «Analyse multimédia interactive du son et des musiques », séance du 18 décembre 2002.

www.ina.fr/grm/outils_dev/theorique/seminaire/semi-2003/semi2_1/inter/AA1.fr.html

BASSET C., Gamelan mécanique, 2003.

webware.cite-musique.fr/www/gamelan/shock.html

Clés d'écoute du Laboratoire d'ethnomusicologie du Musée de l'Homme

www.ethnomus.org/ecoute/

Film

ZEMP H., TRAN QUANG HAI, Le chant des harmoniques (The Song of Harmonics), 16 mm, 38 min, coproduction CNRS Audiovisuel et Société Française d'ethnomusicologie, 1989. 